\title{
Carboxypeptidase B inhibits complement and cartilage loss
}

"Carboxypeptidase B plays a protective role in osteoarthritis by inhibiting formation of the complement membrane attack complex, and potentially through additional anti-inflammatory activities," asserts William Robinson, introducing his latest research published in Arthritis \& Rheumatism. The new study adds to our understanding of protective and pathogenic mechanisms in this disease.

Carboxypeptidase B (CPB) cleaves $\mathrm{C}$-terminal basic residues from various substrates, including fibrin and several proinflammatory proteins. "We previously demonstrated that $\mathrm{CPB}$ has a critical role in downregulating inflammatory responses in rheumatoid arthritis," explains Robinson. "My lab's

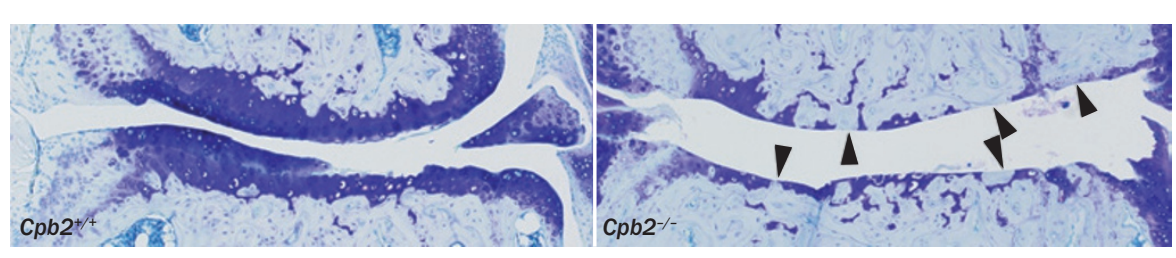

CPB-deficient $\left(\mathrm{Cpb2}^{---}\right)$mice exhibit exacerbated OA in the destabilization of the medial meniscus model. Courtesy of W. Robinson. overarching hypothesis is that low-grade inflammation contributes to osteoarthritis (OA) pathogenesis, and so we wanted to determine whether CPB might also play a part in OA."

The researchers used a mouse medial meniscectomy model of OA to explore the effects of CPB deficiency on disease development. 16 weeks after surgery, CPB-deficient mice displayed exacerbated cartilage loss and synovitis compared with wild-type mice, implicating CPB as a protective mediator. Examining its relevance in humans, the investigators found that high proCPB concentrations in the synovial fluid of patients with OA were associated with elevated levels of proinflammatory cytokines, such as IL- $1 \beta$, and complement components, suggesting that CPB is induced by a feedback loop to downregulate inflammation in the joint.

So, how does CPB mediate such effects? The complement system has been previously linked to OA pathogenesis, and preincubation with $\mathrm{CPB}$ diminished the ability of human serum to induce the formation of soluble membrane attack complexes. Moreover, CPB inhibited complement-induced cell lysis in vitro.

Thus, the authors suggest that a CPBmediated reduction in complement activation might limit OA-associated joint damage. "We now want to better define both the mechanisms by which CPB exerts its protective functions and the role of $\mathrm{CPB}$ in various clinical subtypes of pre-OA," concludes Robinson.

Isabel Woodman

Original article Lepus, C. M. et al. Carboxypeptidase B serves as a protective mediator in osteoarthritis. Arthritis Rheum. doi:10.1002/art.38213 